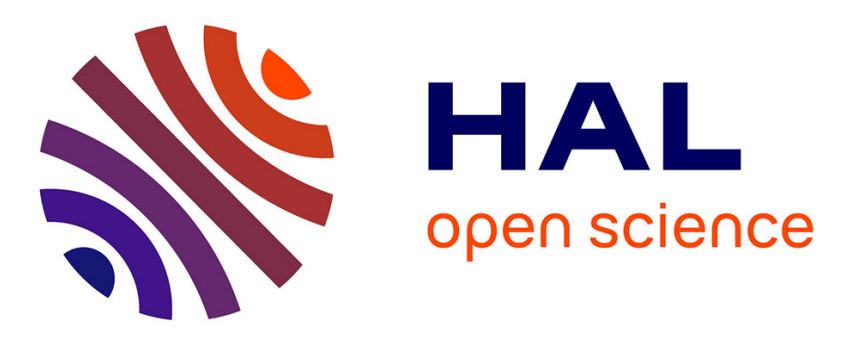

\title{
Evolution of 3C Cyber-Physical Systems Architecture for Industry 4.0
}

Ahmadzai Ahmadi, Ali Hassan Sodhro, Chantal Cherifi, Vincent Cheutet, Yacine Ouzrout

\section{- To cite this version:}

Ahmadzai Ahmadi, Ali Hassan Sodhro, Chantal Cherifi, Vincent Cheutet, Yacine Ouzrout. Evolution of 3C Cyber-Physical Systems Architecture for Industry 4.0. 8th Workshop on Service Orientation in Holonic and Multi-Agent Manufacturing (SOHOMA 2018), Jun 2018, Bergame, Italy. hal-01788468

\section{HAL Id: hal-01788468 \\ https://hal.science/hal-01788468}

Submitted on 12 Dec 2018

HAL is a multi-disciplinary open access archive for the deposit and dissemination of scientific research documents, whether they are published or not. The documents may come from teaching and research institutions in France or abroad, or from public or private research centers.
L'archive ouverte pluridisciplinaire HAL, est destinée au dépôt et à la diffusion de documents scientifiques de niveau recherche, publiés ou non, émanant des établissements d'enseignement et de recherche français ou étrangers, des laboratoires publics ou privés. 


\title{
Evolution of 3C Cyber-Physical Systems Architecture for Industry 4.0
}

\author{
Ahmadzai AHMADI, Ali Hassan SODHRO, Chantal CHERIFI, Vincent CHEUTET, \\ Yacine OUZROUT
}

University Lyon, University Lumière Lyon2, INSA Lyon, DISP EA4570, France

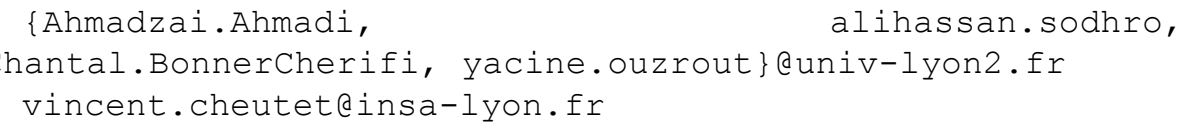

\begin{abstract}
Cyber-Physical System (CPS) is considered as the emerging component for Industry 4.0, and the state-of-the art and standard CPS architecture playing the major role to understand the nature of the industrial landscape. The key problem with traditional CPS architectures is that they are not up-to-mark and convincing to fulfill the needs of smart industries and merely considering vertical and horizontal integration with three components (i.e., human, cyber and physical components) integration, but lacking to adopt, discuss and highlight the key interfacing elements which are very vital for Industry 4.0. So, to remedy these problems and bridging the gap this paper proposes to enhance the 3C CPS architecture based on the traditional $3 \mathrm{C}$ one for industry 4.0 by adopting the main interfacing elements such as connectors, protocols, and sub elements, for example, human, cyber and physical parts. Hence, it can be said that the proposed enhanced $3 \mathrm{C}$ CPS architecture is playing a significant role and will be considered as a guideline and application for future smart manufacturing CPS systems and industries.
\end{abstract}

Keywords: CPS, Industry 4.0, CPS Architectures, Connectors, Protocols

\section{Introduction}

Cyber-physical systems (CPSs) are the emerging and evolutionary paradigm and is the combination of cyber and physical components. CPS is the key interconnecting entity to create the strong ties between physical (e.g. through sensors, actuators, robotics, and embedded systems) and imaginary worlds while exchanging/processing the important and sensitive information. The term CPS was first appeared in 2006, when an NFS workshop was held in Austin, Texas, USA. It was defined as "a system composed of collaborative entities, equipped with calculation capabilities and actors of an intensive connection with the surrounding physical world and phenomena, using and providing all together services of treatment and communication of data available on the network." [1]. CPS is one key technology for industry 4.0 and CPS has attracted a lot of research attention recently about smart manufacturing [2]. 
In order to integrate them inside a production system, a clear purpose and methodology should be defined in order to support manufacturers to get the complete guide. Up to now there is no efficient and effective technique to use the CPS for the smart industry, so keeping this huge demand in mind this paper proposes the enhanced architecture of 3C CPS architecture by introducing the additional ingredients and sub-interfacing components for the better production system. In addition, this is the initial and important step to think and adopt the sub-interfacing components. In the literature there are several different CPS architectures (5C architecture, 8C architecture and 3C architecture) for smart manufacturing. The objective of this paper is to analyze these architectures and propose the most suited to the needs of CPS and Industry 4.0.

This paper proposes the enhanced 3C CPS architecture based on the traditional 3C by introducing the main interfacing elements i.e., connectors, protocols, and sub-elements, e.g., human, cyber and physical parts. Besides, the adaptation of sub-interfacing elements improves the standardization level of human, cyber and physical components for Industry 4.0. Moreover, the practical usage of our proposed CPS architecture will be in the embedded system based electronics industries.

Remaining of the paper is organized as follows. Section 2 presents the rigorous related works of CPS architectures and discusses the conventional CPS architecture for Industry 4.0. Section 3 proposes the enhanced 3C CPS architecture for smart manufacturing Industry. Paper is concluded in the Section 4.

\section{$2 \quad$ Related Works}

\subsection{C CPS Architecture for Industry 4.0}

The 5C architecture is proposed by [3], to build the CPS for smart factories. The 5C architecture consists of 5 levels; from bottom to top, Connection, Conversion, Cyber, Cognition, and Configuration (Fig.1). The connection level is concerned with obtaining the accurate and reliable measurement of the production machines by connecting sensors to machines. Recently communication protocols have been developed to allow users to communicate and collect the signals from various types of controller, such as MT Connect. An example used MT Connect data from machine tools for process planning verification. The conversion level pays attention to the conversion of the measurement data into information. It has received considerable attention specifically for prognostics and health management (PHM). The cyber level emphasizes that more information can be obtained through connecting more machines. It utilizes the concept of time machine to describe every physical entities' twin model in the cyber space. The cognition level provides users with the information to assist them to make decisions. It is meant to use decision making and reasoning methods to provide appropriate actionable suggestions to the operators and business managers. The configuration level finally gives feedback back to the physical system [3]. It enables the machines with self-adjusting and selfconfiguration capability.

An evolution of this architecture has been proposed in [4] with the $8 \mathrm{C}$ architecture, achieved by adding $3 \mathrm{C}$ facets into the $5 \mathrm{C}$ architecture. The $3 \mathrm{C}$ facets are coalition, cus- 
tomer, and content. The coalition facet focuses on the value chain integration and production chain integration between different parties in terms of the production process. The customer facets focuses on the role which the customers play in the production process. The content facet focuses on the extracting, storing, and inquiring the content of product traceability.

\subsection{ACPS Architecture}

The Anthropocentric Cyber- Physical System (ACPS) reference model was defined to provide the highest abstraction level for the Anthropocentric Cyber- reference Architecture for Smart Factories (ACPA4SF) definition [5]. ACPS is a reference model for factory automation that integrates the physical component (PC), the computational/cyber component (CC) and the human component (HC). The basic decomposition abstraction serves to naturally reflect the multiple and context sensitive loci of control for cyber-physical production systems. ACPS reference model is supported by the latest technological developments in service oriented architectures (SOA), semantic Web, human-machine interaction (HMI). The key characteristic of an ACPS reference model is its unified integrity which cannot be further decomposed into smaller engineering artefacts without losing its functionality. From the engineering stance, the ACPS concept emphasizes the adaptive and dynamic division of labor among the ACPS components as a result of their continuous interactions [5]. It tries to capture the common ground encompassing the meaning of CPSs and to identify the core relationships among its composite entities. The ACPS reference model goes beyond the classical architecture of a CPS that simply embeds the human-machine interface in a mechatronic device.

Therefore, the ACPS reference model for factory automation integrates the PC, the $\mathrm{CC}$ and the HC as shown in Fig.2. The relations between these components are usually made via adaptors, optional in many cases, which translate the signals into the specific format of the interacting component. For example: between the PC and $\mathrm{HC}$ there are special displays or meters to measure the working parameters of a ma-chine; between the $\mathrm{CC}$ and $\mathrm{HC}$ there are the classical human-computer interaction (HCI) devices (e.g. screens, mouse, keyboard, etc.), and between the PC and CC there are special transducers or analog-to-digital converters. These components are connected outside the ACPS to their specific dimension: physical (e.g. via mechanical slots), computational (e.g. via computer-specific communication standards), and social (e.g. via natural language). It does not exclude the mediated interactions be-tween the ACPS components (HC to CC via $\mathrm{PC}$, and so on) in the so called "smart environment", when the components will play an active role in shaping these hybrid interactions. The ACPS components participate on a role-basis in an ACPS and their inclusion in a concrete instance depends on the engineering compromises that should be accommodated in its real implementation. A role implements one or more interaction protocols or methods that accommodate multiple adaptation loops between: PC-HC, HC-CC, and PC-CC [5]. 


\subsection{C CPS and ACPS Architecture Comparison}

The main difference between 5C and ACPS architectures for Industry 4.0 is of key parameters such as, $5 \mathrm{C}$ is summarized by 5 levels (connection, conversion, cyber, cognition, and configuration levels), whereas ACPS architecture is described by 3 components (human, cyber, and physical).

In addition, the $5 \mathrm{C}$ architecture emphasized more on vertical integration and less on horizontal integration and has been improved by $3 \mathrm{C}$ components (Coalition, Customer, and Content) [4], while the ACPS architecture emphasis more on human, cyber, and physical components. But they do not consider the main interfacing components while establishing the smart manufacturing plat-form for Industry 4.0. So in order to remedy that problem this section presents the strong sustainable connection between the heterogeneous components in smart manufacturing industries. Besides, the notion of very vital and main interconnecting/interfacing parameters such as, connectors and protocols with proposed enhanced 3C CPS architecture is introduced.

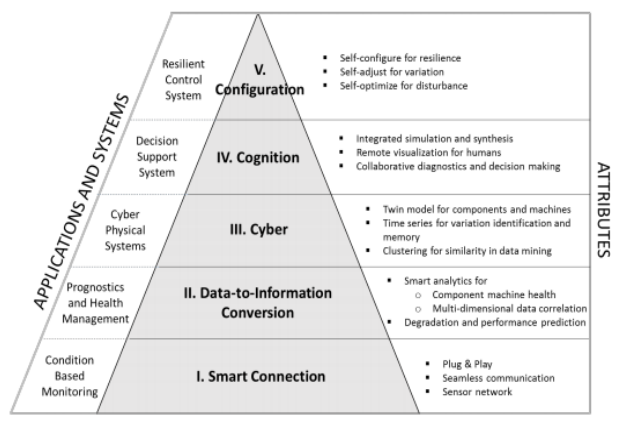

Fig. 1. 5C CPS Architecture [3]

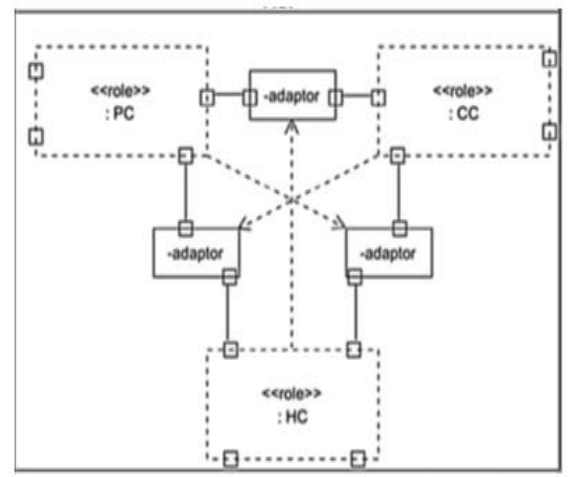

Fig. 2. ACPS Architecture [5]

\section{$3 \quad$ Proposed 3C CPS Architecture for Industry 4.0}

The primary thoughts of control engineers are to improve the performance of the unmanned and manual system based factories because of their complex and engineering procedures and infeasible strategic plan. Therefore, ACPS is considered as an important element for future manufacturing industry [5]. In the ACPS model, the authors proposed all connected Physical Component (PC), Cyber Component (CC), and Human Component (HC) in every individual level of operations. Another model addresses the connected CC and PC managed by human [6]. After all, the context of CPS for manufacturing in Indus-try 4.0 can be observed in three components: 1) Human Component (HC), 2) Cyber Component (CC), and 3) Physical Component (PC). Moreover, the interfaces of HC-CC, CC-PC, and HC-PC play an important role in connecting all components to be-come a united system to attain a common goal describes the components of CPS in manufacturing Fig 3. Smart products are physical products which are equipped with embedded systems, sensors and actuators. Smart product has three core 
elements: Physical components (mechanical and electrical/electronic parts), smart components (control systems, sensors, microprocessors, data storage, software, and user inter-face), and connectivity components (ports, antenna, and wired or wireless interface protocols). In cyber- physical production systems, where the human factor will increasingly play a significant role, there is a clear need to consider humans as endogenous interacting components within a CPS.

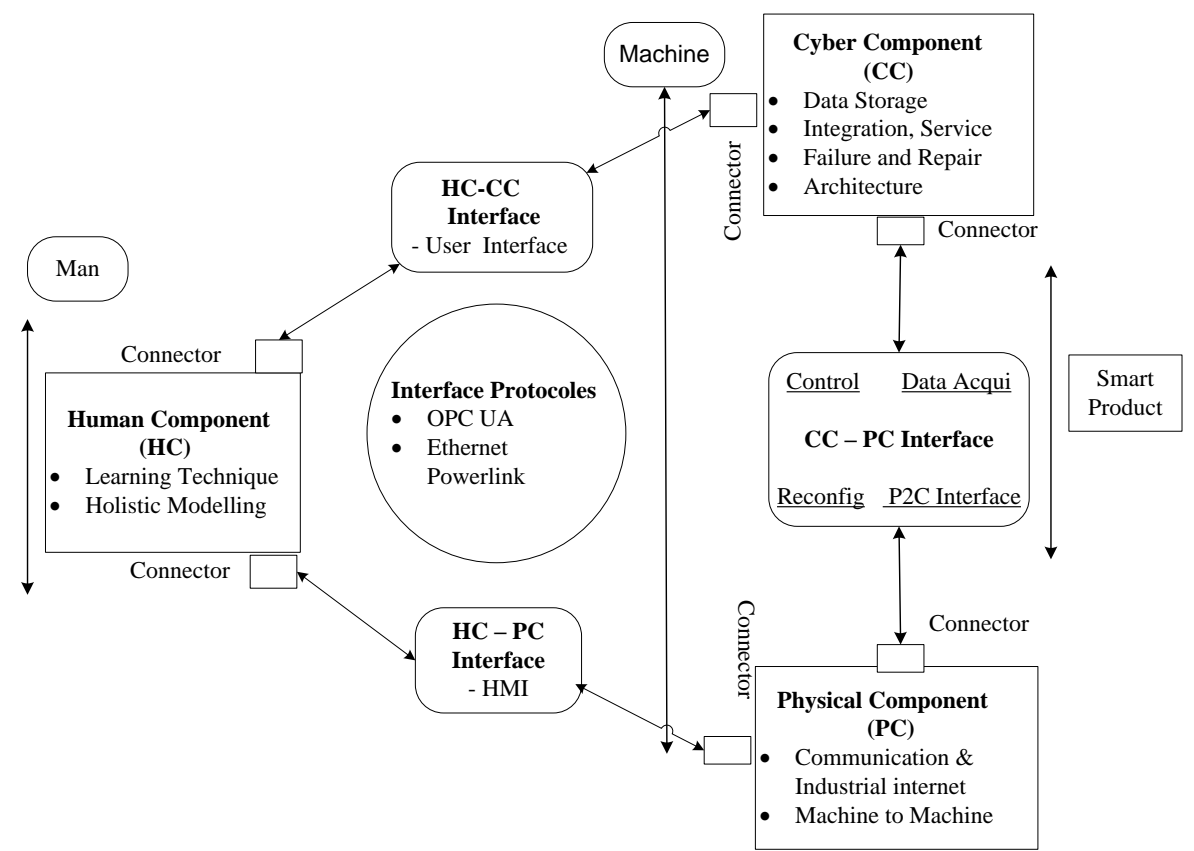

Fig. 3. Proposed 3C CPS Architecture for Industry 4.0

\subsection{Human Component}

Due to the increasing demands of customers for customized products and the rapid changes in machinery/systems of CPS-based manufacturing in Industry 4.0, the production process needs shorter product life cycle, personalized products, and quick employee adaptation to the newly innovative changes [7]. Human is considered as one of the inevitable components for the newly coined Industry 4.0 to be successful, and this component is called as Human Component (HC) [4]. Different articles focus on the HC constituents like holistic Production model or anthropocentric processes and learning techniques to adapt employee to the rapidly changed machinery/systems in the manufacturing industry [4-8].

\subsubsection{Learning Techniques.}


The replacement of the traditional components by dynamic and intelligent CPS demands broader skill of human production workers. Therefore, quicker learning techniques for workers become essential [8]. Industry 4.0 supports work-based learning and an Augmented Reality (AR) based interactive instruction manuals for quicker understanding of changes in production.

\subsubsection{Anthropocentric Model.}

In an Anthropocentric Cyber-Physical Systems (ACPS) for the future manufacturing industry, Human Component (HC) is considered as an important element [5]. The holistic or human model comprises of the cloud assisted service oriented architecture, or a human-centered modelling approach on generic coordination to enable CPSs to integrate human intelligence [6].

\subsection{Cyber Component}

Cyber is another key entity of CPS for manufacturing in Industry 4.0 that uses computing devices as important processing tools. Different articles focus on the CC constituents like data storage, data management and services, failure and repair management for dynamic reconfiguration, and over- all cyber architecture [9-15].

\subsubsection{Data Storage.}

A federative, secure, and cloud-based [9] Virtual Fort Knox platform [10], cloudintegrated CPS (CCPS), and cloud-assisted Service Oriented Architecture (SOA) are used for efficient data storage in collaborative processing.

\subsubsection{Data Management and Services.}

The three-stage model for optimization of production system and sustainable production systems have been used for data integration. The machine learning technique into CPS can bring self-predict capabilities, whereas industrial software-product-service systems (ISPS) in a value chain, Sense- Compute-Control (SCC) applications, and new standard architectures for industrial networks and middleware [11] are popular services.

\subsubsection{Failure and Repair Management.}

Failure management has always been crucial to seamless serial operations. Intelligent ramp-up assistant module and embedded model based advanced production control [12] are used for failure management. Also repair management comprises of the adaptive repair process chain for manufacturing, intelligent predictive maintenance (IPdM) system for reaching Zero-Defect Manufacturing (ZDM), and cloud-based framework for lean MRO of the equipment [13].

\subsubsection{Cyber Components (CC) Architecture.}


Several cyber component architectures are focusing on manufacturing Internet of Things with layered technologies [14], plant engineering, monitoring and diagnosis of Cyber-Physical Systems, organizing and locating services, lifecycle model of CyberPhysical Systems in inter-organizational value networks, and Advanced Planning Systems (APS). Multi-agent system (MAS) architecture has been introduced in the holonic concept of manufacturing, GRACE multi-agent system for integrating process and quality control [15] in the CPS. Moreover, digital description of future production system, intelligent manufacturing using CCPS for Complex Industrial Applications (CIA), multiple resolution models of a manufacturing cell, and Supervisory Control and Data Acquisition (SCADA) systems [16] are the key entity of CPS.

\subsection{Physical Component}

Physical Component (PC) is the lower level hardware part of CPS for manufacturing that uses physical or hardware as a technology component. Different articles focus on the PC constituents like communication and machine-to-machine (M2M) interaction [17-22].

\subsubsection{Communication.}

Communication among constituents of physical components are eminent. CyberPhysical Sensor System (CPSS) in industrial application [17], secure hardware module, and Control automation systems address communication context [18]. As industrial internet has got too much attention due to the boom in the wireless technologies especially, industrial IoT, big data and sensor based techniques [19-21].

\subsubsection{Machine to Machine Interaction.}

Machine-to-Machine (M2M) interaction is important in integrated manufacturing using CPS. Moreover, M2M interaction is also addressed in Industrial Cyber-Physical Systems for Smart-M3 (Multi- vendor, Multi-device, and Multi-part) based two robots interaction and industrial equipment focus on [22].

\subsection{Interfaces}

Different major components (HC, $\mathrm{CC}$, and $\mathrm{PC}$ ) are often integrated through their interfaces: HC-CC, CC-PC, and HC-PC interfaces [23-29].

\subsubsection{HC-CC Interface.}

User Interface. User interface usually plays an important role in bridging human and cyber components. An interface to $\mathrm{CC}$ for self-organized scheduling of labor times in production provides flexible labor utilization. Administrative shells of Industry 4.0, Subject-oriented suggestions for Redesign of Factory (SURF) workplaces, visual computing of ICT solutions in Manufacturing [23], business model based Cyber-Physical 
Production Systems (CPPS) in a value chain, and a UML prole for IoT (UML4IoT), are named a few to address user interfaces [24].

\subsubsection{CC-PC Interface.}

Data Acquisition. In data acquisition, data processing requirements have been analyzed for Industry 4.0. Semantically-enabled plug-and produce and RDF transformation of Automation ML for automated classification of technical specification play a vital role in data acquisition. Delay-aware Mobile wireless sensor network (MWSN) routing protocol and enterprises sensing establishment are also used for this purpose in Industry $4.0[25]$.

\subsubsection{Hardware Control.}

In the seamless component integration, cyber component controls physical components using software automation for Cyber Physical Production Systems (CPPS), the simulation based optimization in (near) real-time, the decentralized self-configuring production control architecture using Configuration and Information Memory (CMory), and software design for cyber-physical automation systems [26].

\subsubsection{Reconfiguration.}

The intuitive robot programming for production industry, robot configuration and programming autonomously, model separation diagnosis of Cyber-Physical Production Systems (CPPS), and reconfigurable smart factory architecture [27] are a few important cyber components to address reconfigurable physical components.

\subsubsection{Physical-to-Cyber Interface.}

Physical-to-cyber interface plays an important role to integrate physical component to cyber component through data communication. We have found different physicalto-cyber interface components, namely data on a RFID transponder attached to different elements, CPS for Industrial Automation Systems (IAS) [28], and trace- ability and tracking in the automotive domain.

\subsubsection{HC-PC Interface.}

Human Machine Interface (HMI). Human-Machine Interface (HMI) is eminent in $\mathrm{HC}$ and PC integration. HMI and data exchange between all modules for machine-tomachine $(\mathrm{M} 2 \mathrm{M})$ and machine-to-human $(\mathrm{M} 2 \mathrm{H})$ communications in production processes are well managed by a Workflow Manager (WFM). Smart glass-based wearable technologies support operators' activities in a CPS manufacturing environment. Moreover, hand-held mobile devices, head mounted display (HMD) and user-context-aware services using model-view-controller environment (MVCE) plays an important role in interfaces. Nevertheless, the self-optimization mechatronics and the social robotics and Human-Computer Interaction (HCI) with Tangible User Interface (TUI) are a few examples of HMI [29]. 


\subsection{Connectors}

Industry 4.0 will bring a dynamic change in the technology as well as the conventional trends [30]. Widely accepted concept of the self-adaptive industrial platform with strong network of the business personals, traders, suppliers and consumers are benefitted at the maximum level. Also with the technological boom and size of the machines and devices is getting smaller, so it is easy for them to exchange ideas and information in a detailed manner. In the meantime Internet of Things and CPS have entirely changed the land-scape of the world with the miniaturized sensor enabled devices which can easily accessed by transferring critical and sensitive information. So, for the sustainable and efficient communication between machines connectors are playing the major role with high capability. For instance, ERNI connectors are so active and intelligent to tackle all the hurdles of present, past and future, and that is why these connectors are the potential candidates in the market with high demand for the Industry 4.0. Besides, most popular connectors for Industry 4.0 are: ERmet 2.0 HM, ERmet ZD, DIN 41612, Micro Speed, Mod Jack RJ45 / RJ11, Erbic field bus interface.

\subsection{Interface Protocols}

Protocols are the building blocks of the efficient and effective communication between devices, machines in the CPS and IoT environment, they adaptively adjust themselves by establishing the strong network. Few of the protocols are discussed as a remarkable ingredients e.g., Profibus/Profinet. OPC-UA Fieldbus, Ethernet Powerlink, for further details see the Table.1 [17, 18]. Fieldbus (Process Field Bus) \& Profinet (Process Field Net) International (PI) and OPC Foundation are playing the key functioning role in an integrated manner since long time. Specifically, exchanging information between machines/devices for instance, operator stations via the controller level or for production data from devices to corporate information technology (IT) sector with specific requirements from several service achievers and providers. Fieldbus and Profinet Inter-national (PI) is the huge automation group in the world and responsible for Fieldbus and Profinet, are considered as the two key technology-oriented players in automation presently [31].

OPC Unified Architecture (OPC UA) is the data transferring standard for safe, reliable, manufacturer and platform-independent industrial communication. It enables data exchange between products from distinct vendors and operating system developers. The OPC UA standard is based on specifications that are developed in association with the manufacturers, users, research institutes and consortia for transferring the reliable and safe information among the heterogeneous networks. The main disadvantage of OPC UA is inappropriate for real-time applications. As Fieldbuses integrates automation products from various distinct manufacturers with standardized technology. This intends to discuss and deploy different tools and interface modules, and limitations on diagnostic and test-bed components [32]. Powerlink is the emerging ingredient in the CPS protocols for further details see the Table 1 . 
Table 1. CPS Protocols and description

\begin{tabular}{|c|c|l|}
\hline Protocols & Standard & \multicolumn{1}{c|}{ Description } \\
\hline Profinet & $\begin{array}{c}\text { IEC 8802, } \\
\text { IEEE 802.3 }\end{array}$ & $\begin{array}{l}\text { Profinet (Process Field Net) is an industry technical standard for data } \\
\text { communication over Industrial Ethernet, designed for gathering infor- } \\
\text { mation from, and monitoring, equipment in industrial systems, with a spe- } \\
\text { cific power in transferring data under critical time bound (on the order of } \\
\text { 1ms or less). }\end{array}$ \\
\hline Profibus & IEC 61158 & $\begin{array}{l}\text { Fieldbus (Process Field Bus) is a standard for fieldbus communication } \\
\text { in automation technology and PROFIBUS is openly published as part of } \\
\text { IEC 61158. }\end{array}$ \\
\hline Fieldbus & IEC 61158 & $\begin{array}{l}\text { Fieldbus systems, just like Internet, apply internationally accepted com- } \\
\text { munications reference model OSI (Open Systems Interconnection) in the } \\
\text { definition of communication functions. Nevertheless, the model consid- } \\
\text { ered in industrial systems is a simplified version with merely three layers } \\
\text { application, data link and physical. }\end{array}$ \\
\hline Ethernet & IEC 61158- & $\begin{array}{l}\text { OPC Unified Architecture (OPC UA) is a machine to machine communi- } \\
\text { cation protocol for industrial automation developed by the OPC Founda- } \\
\text { tion }\end{array}$ \\
\hline Powerlink & IEC 62541 & $\begin{array}{l}\text { Ethernet POWERLINK is a protocols for exchanging information among } \\
\text { industrial devices intends at several devices, machines and equipment. It } \\
\text { is designed with the key target of being deployed from the machine level } \\
\text { to the process level, always involving industrial plant communications. }\end{array}$ \\
\hline
\end{tabular}

\section{Conclusion and Future Work}

Cyber physical system is playing an important and remarkable role in the design and development of future manufacturing industries. CPS is the paradigm shift with significant contribution Industry 4.0. In this paper, we have compared 5C CPS and ACPS architectures, then proposed the 3C CPS architecture to strengthen the CPS-enabled smart manufacturing in Industry 4.0. The proposed architecture is based on the ACPS architecture consists of 3 components, namely the human, cyber, and physical levels. In addition to the ACPS 3 levels, the proposed architecture adopts sub-elements of ACPS architecture and interfacing parameters such as, connectors, and protocols. Hence, it can be said that the proposed enhanced 3C CPS architecture playing a significant role and considered as a guideline for future smart manufacturing systems.

In near future we will focus on challenges to CPS for manufacturing. Standardization is one of the key problem and element of smart manufacturing, because it play a critical role in ensuring the safety, quality and reliability of products, processes and services; efficient production; cost reduction through competition; supporting regulation. We need more and more work on standardization for maturing this new emerging technology [33]. Further the several future trends will be considered and adopted with the proposed 3C CPS architecture to develop the standard prototype for the smart manufacturing system as an emerging application for industry 4.0. Finally, the proposed CPS architecture will be applicable for embedded system based electronics industries. 


\section{$5 \quad$ Acknowledgment}

This research work has been supported by SmartLink Project, Erasmus Mundus Program. The authors would like to thank SmartLink Project and Universite Lumiere Lyon2.

\section{References}

1. Sadiku, M. N., Wang, Y., Cui, S., \& Musa, S. M. (2017). Cyber-Physical Systems: A Literature Review. European Scientific Journal, ESJ, 13(36).

2. Lu, Y. (2017). Industry 4.0: a survey on technologies, applications and open research issues. Journal of Industrial Information Integration, 6, 1-10.

3. Bagheri, B., Yang, S., Kao, H. A., \& Lee, J. (2015). Cyber-physical systems architecture for self-aware machines in industry 4.0 environment. IFAC-PapersOnLine, 48(3), 1622-1627.

4. Jiang, J. R. (2017, May). An improved Cyber-Physical Systems architecture for Industry 4.0 smart factories. ICASI, 2017 International Conference on (pp. 918-920). IEEE.

5. Pirvu, B. C., Zamfirescu, C. B., \& Gorecky, D. (2016). Engineering insights from an anthropocentric cyber-physical system: A case study for an assembly station. Mechatronics.

6. Thramboulidis, K. (2015). A cyber-physical system-based approach for industrial automation systems. Computers in Industry, 72, 92-102.

7. Ali Hassan Sodhro (2018), Convergence of IoT and Product Lifecycle Management in Medical Health Care, Future Generation Computer Systems: Special Issue on Emerging Edge-of-Things Computing: Opportunities and Challenges, Elsevier.

8. Hummel, V., Hyra, K., Ranz, F., \& Schuhmacher, J. (2015). Competence development for the holistic design of collaborative work systems in the Logistics Learning Factory.

9. Ali Hassan Sodhro, Arun Kumar, Gul Hassan Sodhro (2018), '5G-based Transmission Power Control Mechanism in Fog Computing for IoT Devices', MDPI Sustainability, 10 (4), 1-17.

10. Holtewert, P., Wutzke, R., Seidelmann, J., \& Bauernhansl, T. (2013). Virtual fort knox federative, secure and cloud-based platform for manufacturing. Procedia CIRP, 7, 527-532.

11. Fang, C., Liu, X., Pardalos, P. M., \& Pei, J. (2016). Optimization for a three-stage production system in the Internet of Things: procurement, production and product recovery, and acquisition. The International Journal of Advanced Manufacturing Technology, 83(5-8), 689-710.

12. Gradišar, D., Glavan, M., Strmčnik, S., \& Mušič, G. (2015). ProOpter: An advanced platform for production analysis and optimization. Computers in industry, 70, 102-115.

13. Spöcker, G., Schreiner, T., Huwer, T., \& Arntz, K. (2016). Programming of adaptive repair process chains using repair features and function blocks. Journal of Computational Design.

14. Cheng, L., Wang, T., Hong, X., Wang, Z., Wang, J., \& Liu, J. (2015). A study on the architecture of manufacturing internet of things. International Journal of Modelling, 23(1), 8-23.

15. Ali Hassan Sodhro, Aicha Sekahri, Yacine Ouzrout (2018), "Energy-efficiency Comparison between Data Rate Control and Transmission Power Control Algorithms for Wireless Body Sensor Networks", International Journal of Distributed Sensor Networks (IJDSN), 14(1), 1-18

16. Toro, C., Barandiaran, I., \& Posada, J. (2015). A perspective on Knowledge Based and Intelligent systems implementation in Industrie 4.0. Procedia Computer Science, 60, 362-370.

17. Berger, C., Hees, A., Braunreuther, S., \& Reinhart, G. (2016). Characterization of cyber-physical sensor systems. Procedia CIRP, 41, 638-643. 
18. Lesjak, C., Ruprechter, T., Haid, J., Bock, H., \& Brenner, E. (2014, September). A secure hardware module and system concept for local and remote industrial embedded system identification. In Emerging Technology and Factory Automation (ETFA), 2014 IEEE (pp. 1-7).

19. Wan, Jiafu, et al. "Software-defined industrial internet of things in the context of industry 4.0." IEEE Sensors Journal 16.20 (2016): 7373-7380.

20. Ali Hassan Sodhro, Ye Li (2013),Medical Quality-of-Service Optimization in Wireless Telemedicine System Using Optimal Smoothing Algorithm",E-Health Telecommunication Systems and Networks (ETSN) Journal, 2(1)

21. Ali Hassan Sodhro, Giancarlo Fortino (2017), Energy Management during Video Transmission in WBSNs", $14^{\text {th }}$ IEEE International Conference on Networking, Sensing and Control (ICNSC), Calabria, Southern Italy, May 16-18.

22. Kashevnik, A., Teslya, N., Padun, B., Kipriyanov, K., \& Arckhipov, V. (2015, April). Industrial cyber-physical system for lenses assembly: Configuration workstation scenario. In Open Innovations Association (FRUCT), 2015 17th Conference of (pp. 62-67).

23. Spath, D., Gerlach, S., Hämmerle, M., Schlund, S., \& Strölin, T. (2013). Cyber-physical system for self-organised and flexible labour utilisation. Personnel, 50, 22.

24. Grangel-González, I., Halilaj, L., Coskun, G., Auer, S., Collarana, D., \& Hoffmeister, M. (2016, February). Towards a semantic administrative shell for industry 4.0 components. In Semantic Computing (ICSC), 2016 IEEE Tenth International Conference on (pp. 230-237).

25. Gölzer, P., Cato, P., \& Amberg, M. (2015, May). Data Processing Requirements of Industry 4.0-Use Cases for Big Data Applications. In ECIS.

26. Niggemann, O., Henning, S., Schriegel, S., Otto, J., \& Anis, A. (2015). Models for Adaptable Automation Software: An Overview of Plug-and-Produce in Industrial Automation. In 11th MBEES Workshop (pp. 73-82),

27. Schmitt, M., Orfgen, M., \& Zühlke, D. (2015). Dynamic reconfiguration of intelligent field devices by using modular software applications. IFAC-PapersOnLine, 48(3), 561-566.

28. Thramboulidis, K. (2015). A cyber-physical system-based approach for industrial automation systems. Computers in Industry, 72, 92-102.

29. Rosenberg, E., Haeusler, M. H., Araullo, R., \& Gardner, N. (2015). Smart Architecture-Bots \& Industry 4.0 Principles for Architecture.

30. Colombo, A. W., Karnouskos, S., Kaynak, O., Shi, Y., \& Yin, S. (2017). Industrial cyberphysical systems, IEEE Industrial Electronics Magazine, 11(1), 6-16.

31. Urbina, M., Astarloa, A., Lázaro, J., Bidarte, U., Villalta, I., \& Rodriguez, M. (2017). CyberPhysical Production System Gateway Based on a Programmable SoC Platform. IEEE.

32. Knezic, M., Dokic, B., \& Ivanovic, Z. (2017). Theoretical and Experimental Evaluation of Ethernet Powerlink PollResponse Chaining Mechanism. IEEE Transactions.

33. Ahmadi, A., Cherifi, C., Cheutet, V., \& Ouzrout, Y. (2017). A Review of CPS 5 Components Architecture for Manufacturing Based on Standards. In SKIMA, International Conference on Software, Knowledge, Intelligent Management and Applications. 